\title{
Adaptabilidade de clones de eucalipto à inoculação de fungos micorrízicos em solo arenoso em São Domingos do Araguaia - PA
}

Antonio Ozenilto de Sousa Lima ${ }^{1}$, Gustavo Schwartz ${ }^{2}$, Arystides Resende Silva ${ }^{2}$, Alexandre Mehl Lunz ${ }^{2}$, Andréa Hentz de Mello ${ }^{3}$, Ulisses Brigatto Albino ${ }^{4}$

${ }^{1}$ Universidade Federal Rural da Amazônia - UFRA. ${ }^{2}$ Empresa Brasileira de Pesquisa Agropecuária - EMBRAPA. ${ }^{3}$ Universidade Federal do Sul e Sudeste do Pará - UNIFESSPA. ${ }^{4}$ Universidade Federal do Pará - UFPA. E-mail: aoslima@gmail.com

\section{Resumo}

Atualmente, as espécies do gênero Eucalyptus figuram nas espécies mais importantes nas plantações florestais comerciais. Estas espécies apresentam dependência de associação micorrízica para o crescimento em condições de campo. Este estudo teve como objetivo avaliar a adaptabilidade de clones de eucalipto sob inoculação de fungos micorrízicos na região de Marabá, sudeste do estado do Pará, Brasil. O experimento foi instalado em delineamento de blocos ao acaso com três repetições, com 12 tratamentos e seis clones. O espaçamento das mudas foi de 3,0 x 3,0 metros, cada parcela com 56 árvores, sendo a metade inoculada com fungos micorrízicos (endomicorrízico e ectomicorrízico). A outra metade das mudas não foi inoculada e funcionou como controle. Cada clone continha 168 indivíduos, totalizando 1008 mudas de clones de eucalipto. A inoculação com isolados micorrízicos não promoveu o maior crescimento em diâmetro e altura dos clones. Os clones: híbrido de Eucalyptus urophylla $x$ Eucalyptus camaldulensis (VM01), de Eucalyptus platyphylla (373) e híbrido de Eucalyptus urophylla x Eucalyptus grandis (1250) apresentaram as menores taxas de mortalidade. Portanto, os clones mais recomendados para a região de estudo foram: híbrido de Eucalyptus urophylla x Eucalyptus camaldulensis (VM01), híbrido de Eucalyptus urophylla $x$ Eucalyptus grandis (A217), Eucalyptus platyphylla (373) e híbrido de Eucalyptus urophylla $x$ Eucalyptus grandis (1250) devido aos seus maiores valores em diâmetro, altura e menor taxa de mortalidade.

Palavras-Chave: inoculação; microbiologia do solo; silvicultura.

Adaptability of eucalyptus clones to the inoculation of mycorrhizal fungi in sand soil in São Domingos do Araguaia - PA

\begin{abstract}
Currently, the species of the genus Eucalyptus figure among the most important species for commercial forest plantations. These species present dependence on mycorrhizal association to grow in field conditions. This study had the objective to evaluate the adaptability of eucalyptus clones under inoculation of mycorrhizal fungi in the Marabá region, southeastern Pará state, Brazil. The experiment was set up in a complete random block design with three replicates, with 12 treatments, and six clones. Seedling spacing was $3.0 \times 3.0$ meters, each plot with 56 trees, where half of them were inoculated with mycorrhizal fungi (endomycorrhizal and ectomycorrhizal). The other half of the seedlings were not inoculated and worked as control. Each clone had 168 individuals, totalizing 1008 seedlings of eucalyptus clones. Inoculation with mycorrhizal isolates did not promote the highest growth in diameter and height of the clones. The clones: hybrid of Eucalyptus urophylla x Eucalyptus camaldulensis (VM01), Eucalyptus platyphylla (373) and hybrid of Eucalyptus urophylla $x$ Eucalyptus grandis (1250) presented the lowest mortality rates. Therefore, the most recommended clones for the study region were: hybrid of Eucalyptus urophylla $x$ Eucalyptus camaldulensis (VM01), hybrid of Eucalyptus urophylla x Eucalyptus grandis (A217), Eucalyptus platyphylla (373), and hybrid of Eucalyptus urophylla $x$ Eucalyptus grandis (1250) due to their highest values in diameter, height, and lower mortality rate.
\end{abstract}

Keywords: inoculation; soil microbiology; silviculture. 


\section{Introdução}

As florestas plantadas desempenham um importante papel produtivo, de recuperação de áreas degradadas e na prestação de serviços ambientais, além de ajudar na redução da pressão de desmatamento e destruição de habitats naturais e biodiversidade. Estas florestas também contribuem no desenvolvimento social e econômico por meio da geração de emprego e renda em toda a cadeia produtiva, promovendo a participação de pequenos e médios produtores rurais (BRITO et al., 2017).

O Brasil apresenta avançado desenvolvimento na área de silvicultura e destaca-se por oferecer o maior programa de reflorestamento do mundo, utilizando principalmente as essências florestais do gênero Eucalyptus (IBÁ, 2017). O sucesso de plantios de eucalipto no setor florestal brasileiro deve-se às altas taxas de crescimento, boa adaptação ecológica, alto rendimento econômico e plasticidade nas propriedades tecnológicas da madeira (DE VECHI; JUNIOR, 2018).

As espécies de eucalipto apresentam dependência de associação micorrízica para seu crescimento e desenvolvimento em condições de campo (LIMA et al., 2014). Nas últimas décadas tem aumentado o reconhecimento da importância funcional e ecológica da simbiose dos fungos micorrízicos não apenas para eucalipto, mas para muitas outras espécies. Estas espécies apresentam grande susceptibilidade à formação de micorrizas, pois esses fungos são micobiontes predominantes em várias plantas comercialmente importantes, dentre as quais se destaca o eucalipto (CAMPOS et al., 2011).

Tanto as micorrizas arbusculares ou endomicorrizas quanto as ectomicorrizas apresentam benefícios aos vegetais e ao meio ambiente. Estes fungos promovem aumento da superfície de absorção e do volume do solo cultivado, levando à melhoria na nutrição e hidratação das plantas, maior tolerância a estresses biológicos e físico-químicos e melhorias no solo por causar maior agregação e estabilidade dos agregados (SMITH; READ, 1997).

Nas ectomicorrizas, as células fúngicas não penetram a parede celular da planta, mas formam duas estruturas: a) uma malha intercelular na epiderme e córtex da raiz denominada rede de Harting, e b) um revestimento derivado desta rede em torno da raiz, denominado manto. Já as endomicorrizas induzem apenas um aumento da ramificação, com raízes maiores e mais profundas, principalmente em habitats com déficit hidríco (FARIA et al., 2017).

As ectomicorrizas e endomicorrizas possibilitam um melhor aproveitamento de água e nutrientes, como $\mathrm{N}, \mathrm{P}$ e $\mathrm{K}$, proporcionando maior resistência ao estresse hídrico, temperaturas elevadas e acidez do solo, além de possibilitar maior tolerância à presença de substâncias tóxicas no solo e a patógenos do sistema radicular (SMITH; READ,1997).

Assim, as ectomicorrizas e endomicorrizas contribuem no estabelecimento e no desenvolvimento das plantas, mesmo em solos pobres ou degradados, apresentando benefícios sobre as plantas não inoculadas na captura de nutrientes, especialmente de P. No caso do eucalipto, as ectomicorrizas e endomicorrizas promovem o estabelecimento a campo de mudas, especialmente em áreas arenosas (MARX et al., 1977).

Sendo assim, considerando a baixa fertilidade dos solos onde vêm sendo implantados os eucaliptos, como é o caso em grande parte do sudeste do estado do Pará, fazse necessária a adoção de alternativas econômicas e ambientalmente viáveis para ampliar a produção das essências destinados ao florestamento dessas áreas.

Uma alternativa é o uso de micorrizas arbusculares e ectomicorrizas para o aumento da absorção de nutrientes e resistência ao ataque de patógenos do solo, melhorando a sobrevivência destas plantas em solos de baixa fertilidade. Nesse sentido, este trabalho teve como objetivo avaliar a adaptabilidade de clones de eucalipto inoculados com fungos ectomicorrizicos e fungos micorrízicos no sudeste do estado do Pará.

\section{Material e métodos}

Área de estudo

O estudo faz parte do projeto Biomas: contribuições para a proteção e uso sustentável das paisagens brasileiras (CNA/EMBRAPA), subprojeto AM05 "Adaptabilidade de diferentes clones comerciais de eucaliptos com e sem inoculação de ectomicorrizas para região sudeste do Pará". A parte experimental do projeto foi instalada na Fazenda Cristalina, município de São Domingos do Araguaia, região de Marabá, sudeste do estado do Pará em janeiro de 2015. A área de estudo localiza-se sob a latitude de 50 36' 6.3" S e longitude 48 29' 2.6" O, em uma área de $11.869 \mathrm{~m}^{2}$. A pecuária extensiva era desenvolvida 
na área de estudo desde a década de 1980 até a instalação do experimento (Figura 1).

Figura 1. Localização da Fazenda Cristalina, São Domingos do Araguaia-PA.

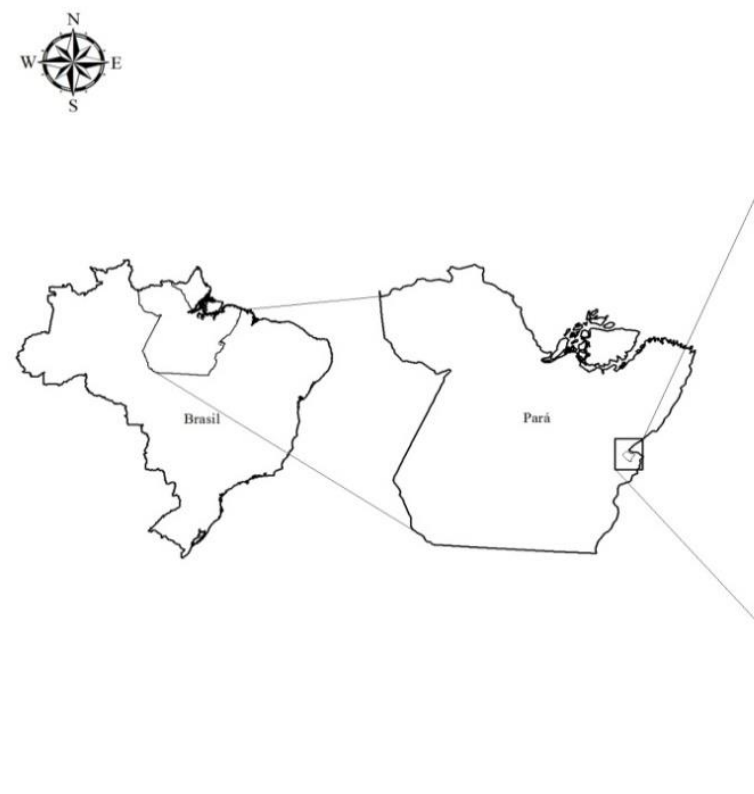

O solo do experimento apresenta baixos teores de carbono, baixa CTC e elevado teor de alumínio trocável e segundo Ramos et al. (2016) foi classificado como Latossolo VermelhoAmarelos Distrófico espessarênico A moderado Textura média relevo plano. O clima da região é tropical semi-úmido (Aw/As) exibindo temperaturas médias mensais entre $22,9{ }^{\circ} \mathrm{C}$ e $32,0{ }^{\circ} \mathrm{C}$, com média anual de $26,0{ }^{\circ} \mathrm{C}$ e

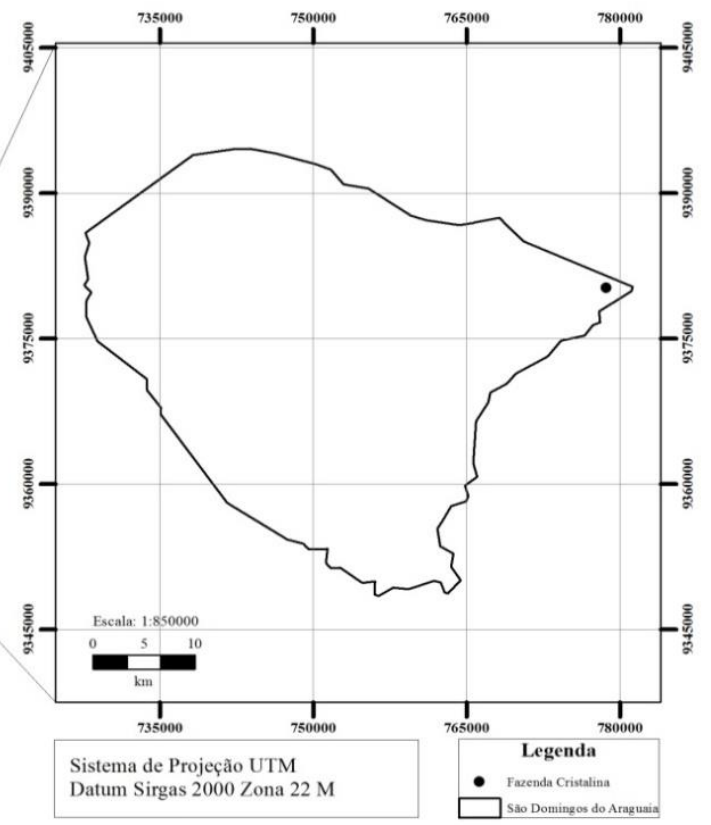

precipitação anual de $1.976 \mathrm{~mm}$. O período mais chuvoso vai de janeiro a março, e o mais seco vai de julho a setembro (RAMOS et al., 2016).

\section{Preparo da área}

Foi realizada uma amostragem de solo para a caracterização dos atributos químicos e granulométricos no ano de 2013 (Tabela 1).

Tabela 1. Características químicas e granulométricas do solo na profundidade $0-20 \mathrm{~cm}$, na fazenda Cristalina em São Domingos do Araguaia-PA.

\begin{tabular}{|c|c|c|c|c|c|c|c|c|c|c|c|}
\hline $\begin{array}{l}\text { Prof. } \\
(\mathrm{cm})\end{array}$ & $\begin{array}{c}\mathrm{pH} \\
\left(\mathrm{H}_{2} \mathrm{O}\right)\end{array}$ & $P$ & $\mathrm{Ca}$ & $\mathrm{Mg}$ & K & $\mathrm{Al}$ & $\mathrm{H}+\mathrm{Al}$ & Carbono & Areia & Silte & Argila \\
\hline & & $\left(\mathrm{mg} \cdot \mathrm{kg}^{-1}\right)$ & & - & . & $m^{-3}$ & ----- & $\mathrm{g} \mathrm{dm}^{-3}$ & $-\ldots$ & $-\mathrm{g} . \mathrm{kg}^{-1}$ & ב--- \\
\hline $0-20$ & 4,8 & 7,4 & 1,3 & 0,5 & 0,1 & 0,2 & 3,2 & 12,4 & 912 & 38 & 50 \\
\hline
\end{tabular}

Análise realizada no laboratório de Solos da Embrapa Amazônia Oriental. $\mathrm{P}=$ Fósforo; $\mathrm{Ca}=$ Cálcio; $\mathrm{Mg}=$ Magnésio; $\mathrm{K}=$ Potássio; Al = Alumínio; $\mathrm{H}+\mathrm{Al}$ = Hidrogênio + Alumínio.

O plantio foi feito com o uso de motocoveadoras e nesta fase realizou-se controle da vegetação espontânea em toda a área experimental com uso de roçadeiras costais e controle de formigas cortadeiras em toda a área e no entorno. Foram aplicadas iscas formicidas granuladas de princípio ativo fipronil, na dose de $8 \mathrm{~g} / \mathrm{m}^{2}$ de terra.

Foi realizada a aplicação de calcário dolomítico na cova (100 g/cova) seguido pelo plantio, onde foram adicionados $400 \mathrm{~g} /$ cova de termofosfato (Yoorin) e $50 \mathrm{~g} /$ cova de NPK (10-28-20) além de 1 litro/planta de hidrogel hidratado, para cada litro utilizou $5 \mathrm{~g}$ de higrogel em pó, onde o mesmo foi hidratado em um período de 45 minutos para que o higrogel possa absorver a água e ficar com aspecto gelatinoso.

Foram feitas três adubações de cobertura: a primeira adubação de cobertura foi feita aos 30 dias após o plantio com 100 $\mathrm{g} /$ planta de NPK (10-28-20) em duas covetas 
laterais localizadas de 10 a $15 \mathrm{~cm}$ da muda, distribuindo metade da dose de cada lado da muda. Repetiu-se o mesmo processo na segunda e terceira adubação de cobertura nos dois anos seguintes.

A irrigação foi realizada a cada 15 dia nos meses mais secos do primeiro ano e a manutenção consistiu no monitoramento de formigas cortadeiras durante todo o período experimental. Realizaram-se capinas químicas com o uso do glifosato ( $\mathrm{N}$-(fosfonometil)glicina) antes da 1a e 2a adubação de cobertura, utilizando a concentração de $2 \%$ do produto, ou seja para cada 20 litros de uma bomba costal colocou $400 \mathrm{ml}$ de glifosato, onde foi aplicado na entrelinha de plantio e na linha entre os eucalipto com o uso do chapéu de napoleão acoplado ao bico do pulverizador para manter o cultivo do eucalipto livre de plantas daninhas, com cuidado para que não ocorresse deriva do produto ou fitotoxicidade. A desrama do eucalipto ocorreu naturalmente, não havendo nenhuma intervenção.

\section{Delineamento experimental}

$O$ experimento foi instalado seguindo o delineamento em blocos ao acaso com três repetições, com 12 tratamentos, seis clones com e sem inoculação de fungos micorrízicos. O espaçamento entre as mudas foi de 3,0 × 3,0 metros. Cada parcela contou com 56 árvores. Foram utilizadas para cada clone 168 mudas, totalizando 1008 mudas de clones de eucalipto (Figura 2).

Figura 2. Croqui do experimento para os testes de inoculação de fungos ecto e endomicorrízicos em clones de eucalipto na fazenda Cristalina, São Domingos do Araguaia-PA.

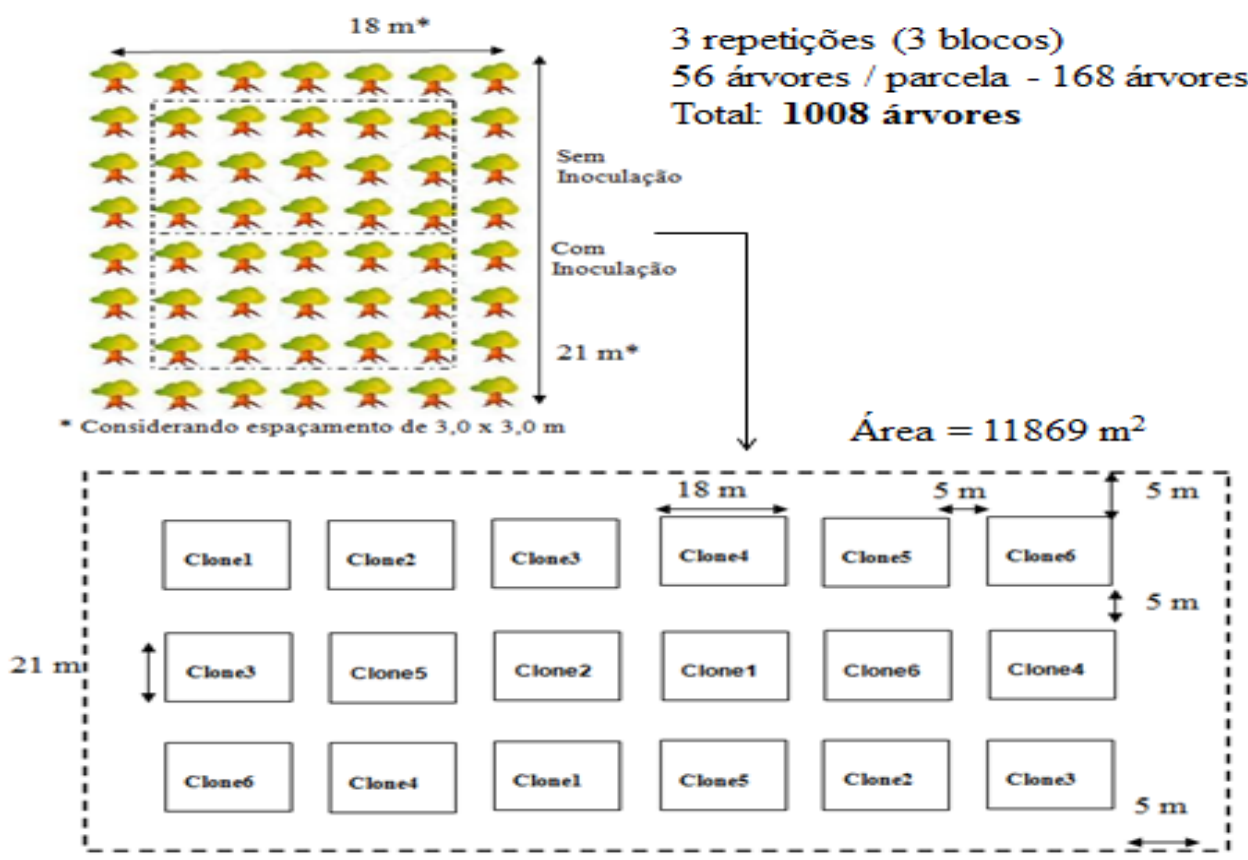

Clone 1, C01253 Eucalyptus urophylla x Eucalyptus grandis; clone 2, VM01 Eucalyptus urophylla $x$ Eucalyptus camaldulensis; clone 3, A211 Eucalyptus urophylla x Eucalyptus grandis; clone 4, A217 Eucalyptus urophylla $x$ Eucalyptus grandis; clone 5, 373 Eucalyptus platyphylla; clone 6, 1250 Eucalyptus urophylla $\times$ Eucalyptus grandis.

Os fungos utilizados para a inoculação consistiram de uma mistura de fungos micorrízicos arbusculares também chamados de endomicorrízicos da espécie Glomus clarum e Glomus etunicatum misturados com o fungo ectomicorrízico Pisolithus microcarpus e a outra metade não foi inoculada. Os fungos foram doados do Banco de Inóculo da Faculdade de Ciências Agrárias de Marabá da Universidade
Federal do Sul e Sudeste do Pará. Eles consistiram de uma mistura de três gramas de esporos e micélios dos respectivos fungos misturados em areia autoclavada, sendo que a inoculação ocorreu no momento do plantio das mudas em cada cova.

Os clones utilizados foram: quatro clones híbridos de Eucalyptus urophylla $x$ Eucalyptus grandis (C01253, A211, A217 e 1250), 
um clone híbrido de Eucalyptus urophylla $x$ Eucalyptus camaldulensis (VM01) e um clone de Eucalyptus platyphylla (373), os quais foram adquiridos junto a viveiros de empresas florestais na região.

\section{Variáveis analisadas}

Foi medido o CAP (Circunferência a altura do peito, a $1,30 \mathrm{~m}$ do solo) e posteriormente transformado para DAP (Diâmetro a altura do peito). Para se medir a altura das plantas foi usado um Vertex. O volume da madeira de cada árvore foi obtido por meio da fórmula: $V=D A P^{2} .(\pi / 4) . h . F F$. Onde: $\mathrm{V}=$ volume de madeira da árvore $\left(\mathrm{m}^{3}\right) ; \mathrm{h}=$ altura total da planta $(\mathrm{cm}) ; \mathrm{DAP}=$ diâmetro a altura do peito $(\mathrm{m}) ; \mathrm{FF}=$ fator de forma $(0,46243)$. $O$ fator de forma utilizado para o cálculo do volume neste estudo foi o mesmo valor desenvolvido por Miranda et al. (2015), para estimar o volume comercial e total de clones de Eucalyptus urophylla $\times$ Eucalyptus grandis, com idade de 30 meses e espaçamento de $4 \times 2 \mathrm{~m}$, em plantio localizado em Nova Canaã do Norte, Mato Grosso. Foi quantificado o número de plantas vivas dos clones de eucalipto e calculada a taxa de mortalidade (relação entre o número de plantas mortas e número de mudas plantadas). As medições foram realizadas aos 180, 360, 720 e 1080 dias após plantio em campo.
Análise dos dados

Os dados foram submetidos à análise de variância e as médias quando significativas comparadas pelo teste de Tukey, ao nível de significância de $5 \%$. As planilhas para formação de banco de dados foram feitas no programa Excel e analisadas com auxílio de programas estatísticos Excel 2010 e Bioestat (AYRES et al., 2007).

\section{Resultados}

Quanto ao efeito da inoculação dos fungos micorrizícos, os clones comerciais de eucalipto apresentaram desempenho semelhante em altura aos 180, 360, 720 e 1080 dias após plantio, com e sem o efeito da inoculação. No entanto, houve diferença significativa no crescimento em altura entre os tipos de clones, o clone híbrido de Eucalyptus urophylla $x$ Eucalyptus camaldulensis (VM01) obteve o melhor desempenho aos 180 dias e aos 360 dias. Aos 720 e 1080 dias o clone que expressou o maior crescimento em altura com e sem o efeito do inoculante foi o clone híbrido de Eucalyptus urophylla $x$ Eucalyptus grandis (A217) em seguida do clone Eucalyptus platyphylla (373), e dos clones híbrido de Eucalyptus urophylla $x$ Eucalyptus grandis (A211, 1250 e CO1253), Os menores valores para esta variável foram observados pelo clone híbrido de Eucalyptus urophylla $x$ Eucalyptus camaldulensis (VM01) (Tabela 2).

Tabela 2. Altura dos clones de eucalipto com e sem inoculação de ectomicorrízas e endomicorrizas aos 180, 360, 720 e 1080 dias, na fazenda Cristalina, São Domingos Araguaia - PA.

\begin{tabular}{cccccc}
\hline \multirow{2}{*}{ Clone } & & \multicolumn{4}{c}{ Altura (cm) } \\
\cline { 3 - 6 } & Tratamento & $\mathbf{1 8 0}$ dias & $\mathbf{3 6 0}$ dias & $\mathbf{7 2 0}$ dias & $\mathbf{1 0 8 0}$ dias \\
\hline \multirow{2}{*}{ VM01 } & Sem inoculante & $1,45 \pm 0,36 \mathrm{a}$ & $1,96 \pm 0,50 \mathrm{a}$ & $8,27 \pm 1,30 \mathrm{~b}$ & $9,50 \pm 1,90 \mathrm{c}$ \\
& Com inoculante & $1,47 \pm 0,39 \mathrm{a}$ & $2,50 \pm 0,57 \mathrm{a}$ & $8,6 \pm 1,57 \mathrm{~b}$ & $10,10 \pm 2,20 \mathrm{c}$ \\
\multirow{2}{*}{ A217 } & Sem inoculante & $1,18 \pm 0,36 \mathrm{~b}$ & $1,57 \pm 0,46 \mathrm{~b}$ & $9,93 \pm 1,61 \mathrm{a}$ & $14,30 \pm 1,30 \mathrm{a}$ \\
& Com inoculante & $1,24 \pm 0,34 \mathrm{~b}$ & $1,69 \pm 0,50 \mathrm{~b}$ & $10,18 \pm 1,43 \mathrm{a}$ & $14,60 \pm 1,20 \mathrm{a}$ \\
\multirow{2}{*}{373} & Sem inoculante & $1,06 \pm 0,22 \mathrm{c}$ & $1,52 \pm 0,32 \mathrm{~b}$ & $8,90 \pm 1,36 \mathrm{~b}$ & $13,30 \pm 1,10 \mathrm{~b}$ \\
& Com inoculante & $1,02 \pm 0,27 \mathrm{c}$ & $1,50 \pm 0,41 \mathrm{~b}$ & $8,47 \pm 1,42 \mathrm{~b}$ & $13,50 \pm 1,40 \mathrm{~b}$ \\
\multirow{3}{*}{1211} & Sem inoculante & $1,02 \pm 0,25 \mathrm{c}$ & $1,40 \pm 0,33 \mathrm{~b}$ & $9,00 \pm 1,73 \mathrm{a}$ & $11,90 \pm 1,90 \mathrm{~b}$ \\
& Com inoculante & $1,04 \pm 0,28 \mathrm{c}$ & $1,50 \pm 0,40 \mathrm{~b}$ & $9,56 \pm 1,61 \mathrm{a}$ & $13,10 \pm 2,00 \mathrm{~b}$ \\
& Sem inoculante & $1,00 \pm 0,29 \mathrm{c}$ & $1,39 \pm 0,36 \mathrm{~b}$ & $7,90 \pm 1,49 \mathrm{~b}$ & $11,90 \pm 1,90 \mathrm{~b}$ \\
& Com inoculante & $1,03 \pm 0,27 \mathrm{c}$ & $1,53 \pm 0,46 \mathrm{~b}$ & $8,04 \pm 1,06 \mathrm{~b}$ & $13,10 \pm 2,0 \mathrm{~b}$ \\
& Sem inoculante & $0,77 \pm 0,21 \mathrm{~d}$ & $1,08 \pm 0,35 \mathrm{c}$ & $7,91 \pm 1,88 \mathrm{~b}$ & $9,50 \pm 1,90 \mathrm{c}$ \\
& Com inoculante & $0,74 \pm 0,25 \mathrm{~d}$ & $1,05 \pm 0,38 \mathrm{c}$ & $7,87 \pm 1,57 \mathrm{~b}$ & $10,10 \pm 2,20 \mathrm{c}$ \\
\hline
\end{tabular}

Médias seguidas da mesma letra na coluna não diferem estatisticamente entre si pelo teste de Tukey $5 \%$. 
Houve diferença significativa para o clone híbrido de Eucalyptus urophylla $x$ Eucalyptus grandis (A211) aos 720 dias para a variável diâmetro, o tratamento com inoculação apresentou maior diâmetro quando comparado ao tratamento sem inoculação. Contudo, aos 720 dias o clone que exibiu diâmetro superior foi o híbrido de Eucalyptus urophylla $x$ Eucalyptus camaldulensis (VM01) seguido do clone híbrido de Eucalyptus urophylla $x$ Eucalyptus grandis (A217) e do clone de Eucalyptus platyphylla (373). Aos 1080 dias o clone que obteve o maior diâmetro foi clone híbrido de Eucalyptus urophylla $x$ Eucalyptus grandis (A217) seguido do híbrido de Eucalyptus urophylla $x$ Eucalyptus camaldulensis (VM01), do clone de Eucalyptus platyphylla (373), e dos híbrido de Eucalyptus urophylla x Eucalyptus grandis (A211 e 1250), sendo que o menor diâmetro foi observado para - clone híbrido de Eucalyptus urophylla $x$ Eucalyptus grandis (CO1253) (Tabela 3).

Tabela 3. Diâmetro a altura do peito dos clones de eucalipto com e sem inoculação de ectomirrízas e endomicorrizas aos 720 e 1080 dias após plantio na fazenda Cristalina, São Domingos Araguaia - PA.

\begin{tabular}{cccc}
\hline \multirow{2}{*}{ Clone } & \multirow{2}{*}{ Tratamento } & \multicolumn{2}{c}{ DAP $(\mathbf{c m})$} \\
\cline { 3 - 4 } & & $\mathbf{7 2 0}$ dias & 1080 dias \\
\hline \multirow{2}{*}{ A217 } & Sem inoculante & $9,34 \pm 1,80 \mathrm{a}$ & $12,95 \pm 2,1 \mathrm{a}$ \\
& Com inoculante & $9,37 \pm 1,85 \mathrm{a}$ & $13,70 \pm 2,0 \mathrm{a}$ \\
\multirow{2}{*}{ VM01 } & Sem inoculante & $8,99 \pm 1,40 \mathrm{a}$ & $12,80 \pm 2,9 \mathrm{a}$ \\
& Com inoculante & $9,37 \pm 1,35 \mathrm{a}$ & $13,05 \pm 3,0 \mathrm{a}$ \\
\multirow{2}{*}{373} & Sem inoculante & $8,52 \pm 1,25 \mathrm{a}$ & $12,26 \pm 1,7 \mathrm{~b}$ \\
& Com inoculante & $8,27 \pm 1,51 \mathrm{a}$ & $12,32 \pm 1,8 \mathrm{~b}$ \\
\multirow{2}{*}{1250} & Sem inoculante & $7,77 \pm 1,45 \mathrm{~b}$ & $11,85 \pm 1,9 \mathrm{~b}$ \\
& Com inoculante & $8,23 \pm 1,35 \mathrm{a}$ & $12,35 \pm 2,0 \mathrm{~b}$ \\
& Sem inoculante & $7,63 \pm 1,44 \mathrm{~b}$ & $11,75 \pm 1,3 \mathrm{~b}$ \\
& Com inoculante & $7,89 \pm 1,08 \mathrm{~b}$ & $11,87 \pm 1,4 \mathrm{~b}$ \\
& Sem inoculante & $7,17 \pm 1,88 \mathrm{~b}$ & $11,30 \pm 2,0 \mathrm{~b}$ \\
& Com inoculante & $7,09 \pm 1,76 \mathrm{~b}$ & $11,71 \pm 1,8 \mathrm{~b}$ \\
\hline
\end{tabular}

Médias seguidas da mesma letra nas colunas não diferem estatisticamente entre si pelo teste de Tukey $5 \%$

Os clones híbrido de Eucalyptus urophylla x Eucalyptus grandis (C01253, A211 e A217) apresentaram as maiores taxa de mortalidade. O clone que apresentou a menor taxa de mortalidade foi o clone híbrido de Eucalyptus urophylla $x$ Eucalyptus camaldulensis (VM01), seguido do clone de Eucalyptus platyphylla (373) e do híbrido de Eucalyptus urophylla x Eucalyptus grandis (1250). Quando comparados o efeito do tratamento (com e sem a inoculação dos fungos) verificou-se que apenas o clone híbrido de Eucalyptus urophylla $x$ Eucalyptus grandis (C01253) apresentou valores bem distintos, com maior taxa de mortalidade para o tratamento sem a inoculação (Figura 3).

Quando comparados os valores do volume estimado a partir do emprego do fator de forma, verificou-se que os clones não apresentaram diferença estatística com e sem efeito do inoculante. No entanto, ao avaliar os diferentes tipos de clones constatou-se que o clone A 217 E. urograndis apresentou maior volume, os demais clones 373 E. platyphylla, 1250 E. urograndis, A211 E. urograndis, C01253 E. urograndis e VM01 E. urocan não diferiram estatisticamente entre si (Figura 4). 
Figura 3. Mortalidade nos clones de eucalipto com e sem inoculação de endomicorrízas e ectomicorrízas aos 1080 dias após plantio na fazenda Cristalina, São Domingos do Araguaia-PA.

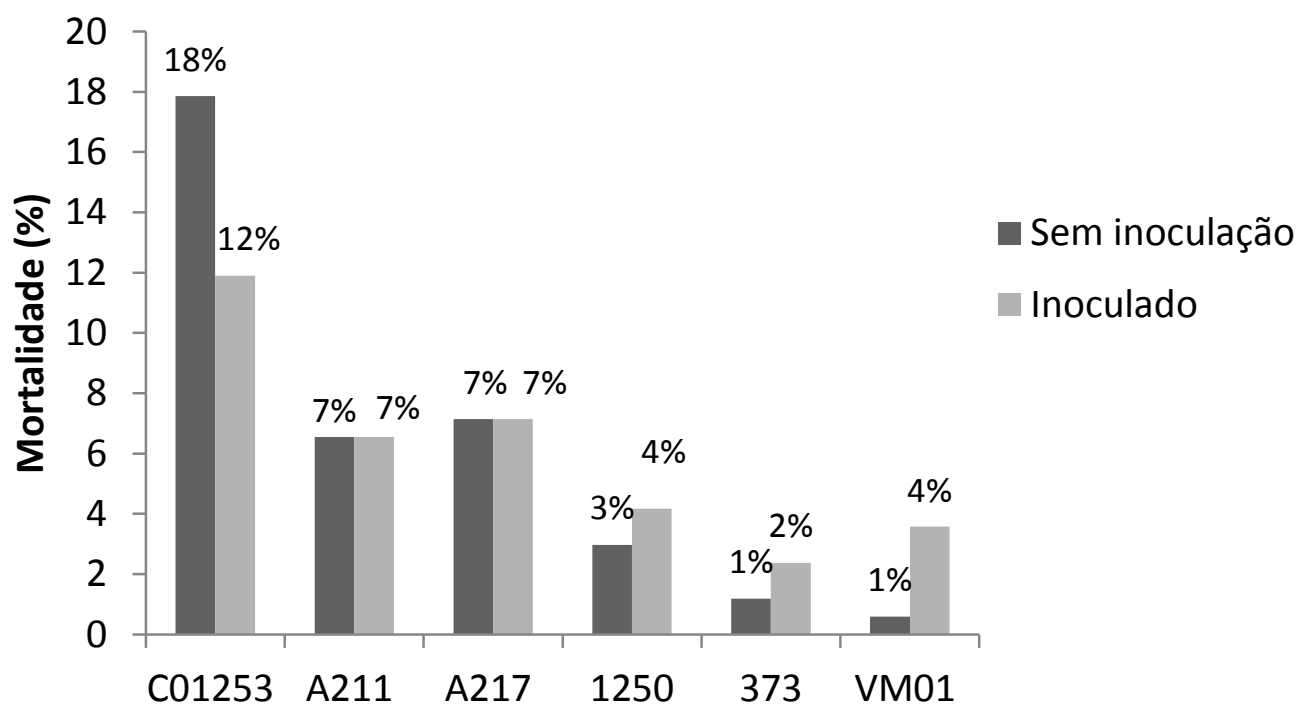

Figura 4. Volume dos clones de eucalipto com e sem inoculação de endomicorrízas e ectomicorrízas na fazenda Cristalina, São Domingos do Araguaia-PA.

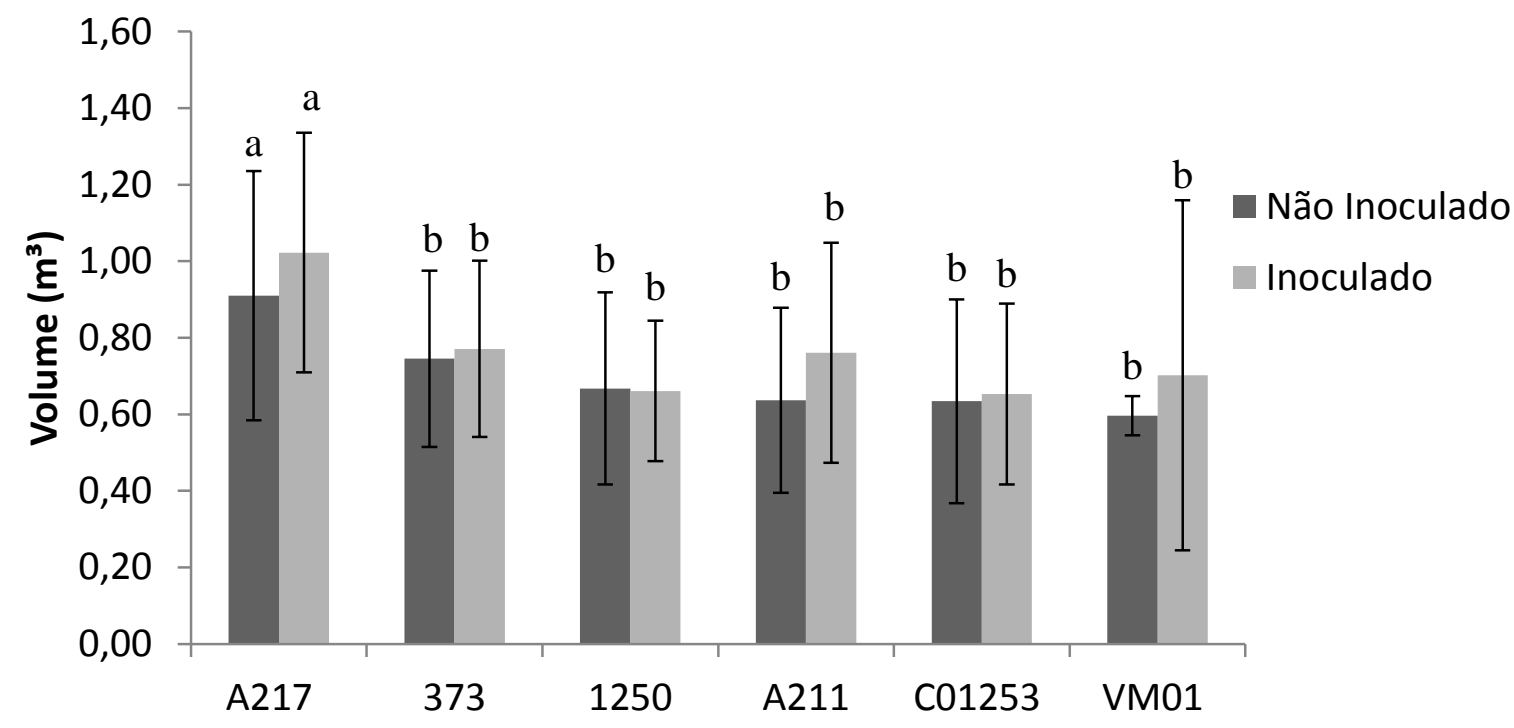

Médias seguidas da mesma letra não diferem estatisticamente entre si pelo teste de Tukey $5 \%$.

\section{Discussão}

Os resultados encontrados neste estudo corroboram com o de Mello (2006) que trabalhou com mudas de Eucalyptus grandis Hillex Maiden inoculadas com o fungo Pisolithus microcarpus em solo arenoso. No estudo foi verificado que as mudas inoculadas produzidas no substrato com fungo e sem fungo não exibiram diferenças quanto à altura, diâmetro e sobrevivência das mudas em campo aos 270 e 360 dias. 0 substrato utilizado no estudo também foi em solo arenoso, bem como os resultados para a altura e diâmetro obtidos foram semelhantes ao encontrados neste estudo.

Trabalhando na produção de mudas de Pinus elliottii Engelm. micorrizadas em solo arenoso em casa de vegetação, Silva et al. (2003) analisaram o comportamento de mudas de pinus inoculadas com fungos ectomicorrízicos, em que os isolados utilizados foram do gênero Pisolithus sp. da região central do estado do Rio Grande do Sul. Constatou-se que os fungos ectomicorrízicos não foram eficientes na absorção de nitrogênio, fósforo e potássio da parte aérea, bem como os valores de altura da planta foram semelhantes 
aos deste trabalho não apresentando diferença significativa quando comparados com e sem o efeito do inoculante. A massa verde e seca da parte aérea não mostrou diferença estatística em relação à testemunha para todos os isolados de inóculos utilizados. Porém, verificou-se que os fungos foram eficientes para as mudas de pinus e proporcionaram maior incremento no comprimento e área superficial específica radicular, sendo importante destacar que neste trabalho não se avaliou as raízes das plantas.

Ferreira et al. (2017) avaliaram o crescimento de espécies de Eucalytpus na região do Médio Paraíba do Sul RJ e obtiveram valores de volume para, híbrido de Eucalyptus urophylla $x$ Eucalyptus grandis, Eucalyptus platyphylla de

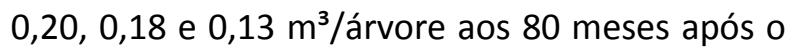
plantio. Neste estudo foi verificado o maior volume para o clone do híbrido de Eucalyptus urophylla $x$ Eucalyptus grandis (A217) com 0,91 $\mathrm{m}^{3}$ /árvore e sem inoculação e $1,02 \mathrm{~m}^{3}$ /árvore sendo que o menor volume foi observado para o clone VM01 E. urocan com 0,60 e 0,70 $\mathrm{m}^{3}$ /árvore com e sem a inoculação dos FMAs e ectomicorrízas. Desta forma, os clones do híbrido de Eucalyptus urophylla $x$ Eucalyptus camaldulensis (VM01), do híbrido de Eucalyptus urophylla $x$ Eucalyptus grandis (A217), clone de Eucalyptus platyphylla (373) e híbrido de Eucalyptus urophylla $x$ Eucalyptus grandis (1250) seriam os mais recomendados para plantio na área de estudo por apresentarem os maiores valores em diâmetro, altura e menores taxa de mortalidade.

Avelar (2016) e Souza (2005) comprovaram que o eucalipto apresenta elevada susceptibilidade à formação de ectomicorrízas que consequentemente promovem seu crescimento através da relação mutualística entre o fungo e planta. No entanto, o que ocorre geralmente é que a atividade dos simbiontes e a associação com as raízes de plantas estão sujeitas a variações nas condições de temperatura, umidade, matéria orgânica do solo, fósforo, nitrogênio, pH e aeração do solo, além de práticas de manejo e procedimentos efetuados durante a formação de mudas (SMITH; READ, 1997).

Sendo assim, os fatores condicionantes ao estabelecimento de cada fungo simbionte na planta e os que influenciam sua eficiência na promoção do crescimento e da adaptabilidade da planta ao ambiente não são totalmente conhecidos. É necessária a avaliação da importância da dupla simbiose para o eucalipto, em vista disso, alguns resultados mostram que pode haver preferência de alguns fungos em colonizarem determinados tipos de solos e plantas hospedeiras (SMITH; READ, 1997).

A utilização de mudas inoculadas em programas de melhoramento pode, contudo, levar à escolha de clones com melhores respostas ao uso de FMA tanto nas condições de viveiro como em campo, como demonstrado por Mello et al. (2009). Ainda assim, os resultados comprovam que os benefícios da simbiose podem variar mesmo entre diferentes clones de eucalipto que exibem semelhante intensidade de colonização (AVELAR, 2016).

No presente estudo, não foi possível detectar diferença estatística significante dos benefícios proporcionados pelo inoculante três anos após o plantio. Possivelmente as micorrizas foram eficientes na manutenção e sobrevivência das plantas no campo. Portanto, devem-se considerar outros fatores, uma vez que as raízes das plantas não foram analisadas. Além disso, os herbicidas utilizados para o controle da vegetação espontânea podem impedir os fungos de desenvolver suas funções não interagindo com o vegetal. Fatores como a quantidade elevada de fósforo no solo da área também pode ter neutralizado por algum tempo os efeitos da associação bem como o tempo de associação que pode ter sido insuficiente para que ocorresse de fato à interação.

\section{Conclusão}

- Os clones de eucalipto não apresentaram diferença estatística para altura e diâmetro aos três anos de idade na região sudeste do Pará. No entanto, os valores médios para altura e diâmetro foram maiores nos clones sob efeito da inoculação.

- O clone híbrido de Eucalyptus urophyllax Eucalyptus grandis (A217) apresentou valores médios superiores para diâmetro e altura no período de três anos após plantio na região do Sudeste do Pará.

- Os clones híbridos de Eucalyptus urophylla $x$ Eucalyptus camaldulensis (VM01), Eucalyptus platyphylla (373) e híbrido de Eucalyptus urophylla $x$ Eucalyptus grandis (1250) apresentaram as menores taxas de mortalidade no período de três anos após plantio na região do Sudeste do Pará. 


\section{Agradecimentos}

Ao CNPq, Embrapa Amazônia Oriental e ao Projeto Biomas: contribuições para a proteção e uso sustentável das paisagens brasileiras (CNA/EMBRAPA), pelo auxílio financeiro e apoio durante a realização da pesquisa.

\section{Referências}

AVELAR, D.C. Doses de inoculante ectomicorrízico em viveiro comercial de mudas clonais de eucalipto. 55 p. Dissertação (Mestrado em Produção Vegetal) - Universidade Federal dos Vales do Jequitinhonha e Mucuri, Diamantina, 2016.

AYRES, M.; JUNIOR, M. A.; AYRES, D. L. (Eds.). Bio estat 5.0. Aplicações estatísticas nas áreas das ciências Bio-médicas. 5. ed. Belém, 2007. 380 p. Disponível em: https://www.researchgate.net/profile/Alex De Assis Dos Santos/publication/263608962 BIOES TAT -

aplicacoes estatisticas nas areas das Ciencias Bio Medicas/links/02e7e53b598e69ebfe000000.pdf. Acesso em: 04 mar. 2019.

BRITO, V. N.; TELLECHEA, F. R. F.; HEITOR, L. C.; FREITAS, M. S. M.; MARTINS, M. A. Fungos micorrízicos arbusculares e adubação fosfatada na produção de mudas de paricá. Ciência Florestal, Santa Maria, v.27, n.2, p.485-497, 2017. https://doi.org/10.5902/1980509827730

CAMPOS, D. T. S.; SILVA, M. C. S.; LUZ, J. M. R.; TELESFORA, R. J.; KASUYA, M. C. M. Colonização micorrízica em plantios de eucalipto. Revista Árvore, Viçosa, v.35, n. 5, p. 965-974, 2011. https://doi.org/10.1590/S0100-

67622011000600002

DE VECHI, A.; JUNIOR, C.A.O.M.; aspectos positivos e negativos da cultura do eucalipto e os efeitos ambientais do seu cultivo. Revista Valore, Volta Redonda, v.3, n.1, p.495-507, 2018. https://doi.org/10.22408/reva312018101495$\underline{507}$

FARIA, A. B. C.; MONTEIRO, P. H. R.; AUER, C. G.; ÂNGELO, A. C. Uso de ectomicorrizas na biorremediação florestal. Ciência Florestal, Santa Maria, v.27, n.1, p.21-29, 2017. https://doi.org/10.5902/1980509826444
FERREIRA, D. H. A.; LELES, P. S. S.; NETO, S. N. O.; PAULA, T. R.; COUTINHO, R. P.; SILVA, R. L. Crescimento e Produção de Eucalipto na Região do Médio Paraíba do Sul, RJ. Floresta e Ambiente, Rio de Janeiro, v. 24, e. 00131315, 2017. https://doi.org/10.1590/2179-8087.131315

LIMA, S. F.; SOUSA, C. S.; Crescimento e nutrição de mudas de clones de eucalipto inoculadas com fungos micorrízicos. Pesquisa Agropecuária Tropical, Goiânia, v. 44, n. 2, p. 110-118, 2014. https://doi.org/10.1590/S198340632014000200006

IBÁ. Indústria Brasileira de Árvores Plantadas. 2017. 80 P. Disponível em: https://iba.org/images/shared/Biblioteca/IBA Re latorioAnual2017.pdf. Acesso em 03 Mar, 2017.

MARX, D. H.; BRYAN, W. C.; CORDELL, C .E. Survival and growth of pine seedlings with Pisolithus ectomycorrhizae after two years on reforestation sites in North Caroline and Florida. Forest Science, Asheville, North Carolina, v. 23, p. 363-373, 1977.

MELLO, A.H. Fungos arbusculares e ectomicorrízicos em áreas de eucalipto e de campo nativo em solo arenoso. Ciência Florestal, Santa Maria, v.16, n.3, p.293-301, 2006. https://doi.org/10.5902/198050981909

MELLO, A.H. et al. Estabelecimento a campo de mudas de Eucalyptus grandis micorrizadas com Pisolithus microcarpus (UFSC Pt116) em solo arenoso. Ciência Florestal, Santa Maria, v.19, n.2, p.149-155, 2009. https://doi.org/10.5902/19805098406

MIRANDA, D. L. C.; JUNIOR, V. B.; GOUVEIA, D. M. Fator de forma e equações de volume para estimativa volumétrica de árvores em plantio de Eucalyptus urograndis. Scientia Plena, Manaus, v. 11, n. 3, p. 1-8, 2015.

RAMOS, M. R.; CURCIO, G. R.; DEDECEK, R. A.; SILVA, A. R. Potencial de uso dos solos: um estudo de caso de São Domingos do Araguaia PA. Revista Amazônia: Ciência \& Desenvolvimento, Belém, v. 13, n. 23, 2016.

SILVA, F. S.; ANTONIOLLI, Z. I.; ANDREAZZA, R. Produção de mudas de Pinus elliottii Engelm. micorrizadas em solo arenoso, Ciência Florestal, 
Santa Maria, v.13, n.2, p.57-65, 2003. https://doi.org/10.5902/198050981742

SMITH, S.E.; READ, D. J.; HARLEY, J. L. Mycorrhizal symbiosis. 2. ed. San Diego, Califórnia: Academic, 1997. $605 \mathrm{p}$.

SOUZA, P.V.D.; CARNIEL, E.; SCHMITZ, J. A. K.; SILVEIRA, S. V. Influência de substratos e fungos micorrízicos arbusculares no desenvolvimento do porta-enxerto Flying Dragon (Poncirus trifoliata, var. monstruosa Swing.). Revista Brasileira de Fruticultura, Jabotical, v.27, p.285-287, 2005. https://doi.org/10.1590/s0100-

29452005000200024 